\title{
Rapid growth of cloud droplets by turbulence
}

\author{
V. Dallas and J. C. Vassilicos \\ Department of Aeronautics, Imperial College, London, SWr 2AZ, UK
}

\begin{abstract}
Assuming perfect collision efficiency, we demonstrate that turbulence can initiate and sustain rapid growth of very small water droplets in air even when these droplets are too small to cluster, and even without having to take gravity and small-scale intermittency into account. This is because the range of local Stokes numbers of identical droplets in the turbulent flow field is broad enough even when small-scale intermittency is neglected. This demonstration is given for turbulence which is one order of magnitude less intense than typically in warm clouds but with a volume fraction which, even though small, is nevertheless large enough for an estimated a priori frequency of collisions to be ten times larger than in warm clouds. However, the time of growth in these conditions turns out to be one order of magnitude smaller than in warm clouds.
\end{abstract}

The rapid growth of warm (ice-free) cloud droplets from $15 \mu \mathrm{m}$ to about $50 \mu \mathrm{m}$ in a short time, typically half an hour, is a well-known phenomenon which still defies explanation. This phenomenon is essential for understanding rain initiation and cloud albedo [1, 2].

Condensation dominates cloud droplet growth till about 10 to $15 \mu \mathrm{m}$ and may be producing a narrow droplet size distribution [1, 3, 4]. If so, a subsequent growth mechanism involving similar-sized particles is required to make the droplet size distribution wide enough for different-sized gravitational collisions to be effective in a final droplet growth mechanism. Such a gravitational settling mechanism may dominate from 30 to 50 micrometres and above [3]. The identification of what triggers the intermediate growth between $15 \mu \mathrm{m}$ to about $50 \mu \mathrm{m}$ and the rate with which it proceeds are major challenges of cloud physics.

Various authors have already proposed that turbulence may be the basis of the intermediate mechanism whereby initially monodispersed droplets can grow via turbulencegenerated collisions [4, 5]. This mechanism is the specific concern of the present paper. It has been suggested that turbulence in clouds may be generating preferential concentrations (clustering) of droplets which would cause a sharp increase in collision and coalescence events and therefore a fast growth of droplet sizes [1, 6, 7]. It has also been suggested that caustics may activate such fast droplet growth [8]. However, measurements suggest [9] that, in warm clouds, the droplet response time $\tau_{p}$ is much too small compared to the smallest (Kolmogorov) time scale $\tau_{\eta}$ for any significant preferential concentration or caustics to be observable and meaningful. Indeed, Saffman \& Turner 10] considered the case where the Stokes number $\tau_{p} / \tau_{\eta}$ is effectively zero but the droplet size is finite and much smaller than the Kolmogorov micro-length-scale $\eta$ (the smallest length scale of the turbulence). They showed that, in this case, droplet sizes do not grow fast enough to explain cloud dynamics and statistics. Their assumption on the droplet size is accurate as $\eta \sim 1 \mathrm{~mm}$ and droplet radius $a_{p} \sim 10^{-2} \mathrm{~mm}$ in clouds before rain initiation and it is reasonable to assume, as they did, that such very small droplets are spherical. However, they also assumed that turbulent velocity gradients are statistically gaussian, and this is known not to be true. Small-scale turbulence is intermittent and the turbulent velocity gradients are increasingly non-gaussian as the Reynolds number increases [11].

The neglect of intermittency may be a significant shortcoming because rare but powerful turbulence events could cause neighbouring droplets to collide and coalesce faster than one would expect from a consideration of the average properties of the turbulence field. These collisions could generate a few large droplets with high momentum which could trigger a chain of successive collisions when travelling and falling fast through the field of much smaller droplets. In principle, such a chain reaction could lead to significant droplet size growth. Kostinski \& Shaw [12] have already argued that rare but powerful events are required for droplet growth, and that these events may have their cause in small-scale turbulence intermittency. Ghosh et al. [13] have argued that such rare but powerful small-scale eddies can also selectively increase settling velocities and thereby further enhance droplet size growth rates.

In this paper we show that turbulence can generate fast droplet size growth without the need for small-scale intermittency and differential gravitational settling, even when droplets are too small to cluster. We place ourselves in a situation close to but different from Saffman \& Turner's [10]. Close in the sense that we assume gaussian statistics of turbulent velocity gradients, spherical droplets of finite size much smaller than $\eta$ and a particle response time $\tau_{p}$ which, as a result, is very small. However our analysis differs from that of Saffman \& Turner [10] in that it takes into account the broad range of local micro-time scales of the turbulence and therefore a broad range of local Stokes numbers in the flow. Nevertheless, all these Stokes numbers are predominantly too small for any reasonable level of clustering to be clearly present.

We follow the approach of Reade \& Collins [14] who used a Direct Numerical Simulation (DNS) of the incompressible Navier-Stokes equations to generate the turbulent velocity field in which they integrated trajectories of very small spherical particles with high mass density. 
They then applied the algorithm of Sundaram \& Collins [15] to simulate droplet collisions and coalescence. However, their DNS was of 3D homogeneous isotropic turbulence without well-defined inertial range $\left(R e_{\lambda}=55\right)$ and the initial Stokes numbers of their droplets ranged between 0.2 and 0.7 which is large enough for clustering to occur (see Fig. 1 in [16] and Fig. 1 in [17]). Instead, our Stokes numbers are initially all well below 0.1 and we opt for a DNS of 2D inverse-cascading turbulence which ensures a wide $-5 / 3$ energy spectrum, much wider than can be achieved by $3 \mathrm{D}$ DNS. Also, it is well known that the velocity gradient statistics of $2 \mathrm{D}$ homogeneous isotropic turbulence are approximately gaussian [18]. In other words, there is no small-scale intermittency in the turbulence we are using.

We endeavour to use a set of conditions as close to real warm clouds as possible within the extent allowed by our computational capabilities. The ratio of the outer to the inner length-scales of our 2D turbulence is at least $L / \eta=110.8$. This is way below the ratio $10^{5}$ in warm clouds, but high enough to have a wide inertial range (see Fig. 1). The rms turbulence velocity $u^{\prime}$ is set at a value which ensures that if our numerical values of $\eta$ and $\tau_{\eta}$ are taken to correspond to $1 \mathrm{~mm}$ and $10^{-1}-10^{-2} \mathrm{~s}$ respectively as in warm clouds, then $u^{\prime}$ is smaller than the usual value in warm clouds which hovers around $1 \mathrm{~m} / \mathrm{s}$. In fact, our value of $u^{\prime}$ is an order of magnitude smaller which makes it harder for our simulations to produce collisions and also allows the adoption of small Stokes numbers, much smaller than 0.1 as in warm clouds, without having to take inordinately small time steps in our timeintegrations.

In warm clouds, the ratio of the droplet's water density to the density of the surrounding air is $\rho_{p} / \rho_{f} \sim 10^{3}$ and the ratio of the mean droplet radius to the Kolmogorov micro-length scale before rain initiation is $\left\langle a_{p}\right\rangle / \eta \sim 10^{-2}$. In our simulations we take $\rho_{p} / \rho_{f}=10^{3}$ and initial $\left\langle a_{p}\right\rangle / \eta=0.0135$ where $2 \pi / \eta$ is taken to be a large multiple of the filter wavenumber $k_{c}$ (see Fig. 1). The larger we chose this multiple to be, the smaller $\left\langle a_{p}\right\rangle$ becomes and the larger the number of droplet trajectories needed to be integrated if we want to keep a realistic droplet volume/area fraction. The droplet volume fraction is $\phi_{V} \sim 10^{-6}$ in warm clouds but we need to set an equivalent area fraction $\phi_{A}$ in our $2 \mathrm{D}$ simulations. We do this by requiring that the geometrical probability of interception along straight lines between two droplets is

The initial particle size distribution in our simulations is a narrow log-normal with a mean particle radius $\left\langle a_{p}\right\rangle=0.0135 \eta$ and a width $\delta a_{p}$ between smallest the same in 2D and 3D. Given a number $N_{2}$ of homogeneously distributed droplets of radius $a_{p}$ in an area $L_{b o x}^{2}$ in $2 \mathrm{D}$ space, a rough estimate of this $2 \mathrm{D}$ probability is $4 \cdot 3 \cdot 2 a_{p} /\left(2 \pi l_{2}\right)=12 a_{p} /\left(\pi l_{2}\right)$ where $l_{2}=\left(L_{b o x}^{2} / N_{2}\right)^{1 / 2}$ is the average distance between droplets (about 4 droplets at a distance $l_{2}$ from each droplet). Given a number $N_{3}$ of homogeneously distributed droplets of radius $a_{p}$ in a volume $L_{b o x}^{3}$ in $3 \mathrm{D}$ space, a rough estimate of this $3 \mathrm{D}$ probability is $6 \pi\left(2 a_{p}\right)^{2} /\left(4 \pi l_{3}^{2}\right)=6\left(a_{p} / l_{3}\right)^{2}$ where

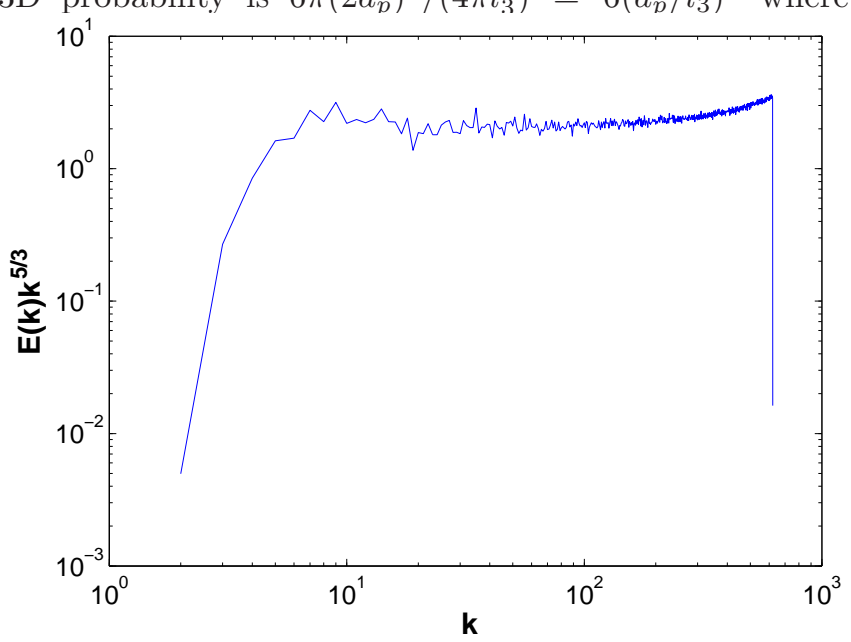

FIG. 1: (Color online) Energy spectrum compensated with $k^{5 / 3}$ and obtained from a $2048^{2}$ inverse-cascading $2 \mathrm{D}$ turbulence using the numerical method and high wavenumber filtering of [19]. The low-pass filtering operation and its wavenumber $k_{c}$ are clearly visible on the right side of the plot. This operation removes the scales directly affected by the smallscale forcing needed to give rise to a stationary turbulence with a $k^{-5 / 3}$ energy spectrum at lower wavenumbers $k$.

it is $l_{3}=\left(L_{\text {box }}^{3} / N_{3}\right)^{1 / 3}$ which is now the average distance between droplets (about 6 droplets at a distance $l_{3}$ from each droplet). Equating the two probabilities yields $\phi_{A}=\frac{\pi}{4}\left(\frac{9 \pi}{16}\right)^{2 / 3} \phi_{V}^{4 / 3}$. Equivalently, this means that the number of particles which we need to immitate a certain $3 \mathrm{D}$ volume fraction $\phi_{V}$ in our $2 \mathrm{D}$ simulations is

$$
N_{2}=\frac{1}{4}\left(\frac{9 \pi}{16}\right)^{2 / 3} \phi_{V}^{4 / 3}\left(\frac{L_{b o x}}{a_{p}}\right)^{2}
$$

Our parameters are tabulated and compared below with the parameters of typical clouds.

and largest size such that $\left\langle a_{p}\right\rangle / \delta a_{p}$ is about 10 or larger (we tried up to 50 and did not find any differences in our conclusions). The Kolmogorov time scale $\bar{\tau}_{\eta}$ is an 


\begin{tabular}{ccccccccccc}
\hline \hline & $N$ & $L / \eta$ & $u^{\prime}$ & $\bar{\tau}_{\eta}$ & $L /\left(u^{\prime} \bar{\tau}_{\eta}\right)$ & $S t$ & $\left\langle a_{p}\right\rangle / \eta$ & $\phi_{V}$ & $\phi_{A}$ & $\rho_{p} / \rho_{f}$ \\
\hline Clouds & - & $10^{5}$ & $1 \mathrm{~m} / \mathrm{s}$ & $0.1 s$ & $10^{3}$ & $10^{-4}-10^{-2}$ & $10^{-2}$ & $10^{-6}$ & - & $10^{3}$ \\
DNS & $2048^{2}$ & 110.8 & 1.24 & 0.0074 & 26.7 & 0.04 & 0.0135 & - & $10^{-4}$ & $10^{3}$ \\
\hline \hline
\end{tabular}

TABLE I: Comparison of parameters in typical warm clouds and in our DNS. The particle response time $\tau_{p}=\frac{2 \rho_{p}}{9 \rho_{f}}\left(\frac{a_{p}}{\eta}\right)^{2} \bar{\tau}_{\eta}$, and we define the Stokes number $S t=\frac{2 \rho_{p}}{9 \rho_{f}}\left(\frac{\left\langle a_{p}\right\rangle}{\eta}\right)^{2}$, where the brackets are now an average over all particles/droplets. (In keeping with $\rho_{p}$ and $\rho_{f}$ which are mass densities in a volume, these expressions for $\tau_{p}$ and $S t$ are for 3D spheres, not 2D disks. It is more important to keep a realistic dependence on $a_{p}$ than $2 \mathrm{D}$ consistency in our model.)

average time scale determined by the average turbulent kinetic energy dissipation rate per unit mass. This average dissipation rate is proportional to $2\left\langle\operatorname{tr}\left(s^{2}\right)\right\rangle$ where $s$ is the strain rate tensor and the brackets are an average over all space. Hence, $\bar{\tau}_{\eta}=1 / \sqrt{2\left\langle\operatorname{tr}\left(\boldsymbol{s}^{2}\right)\right\rangle}$. The local micro-time scales $\tau_{\eta}$ of the turbulence are determined in the same way but in terms of the local turbulent kinetic energy dissipation rates per unit mass which are proportional to the local $2 \operatorname{tr}\left(s^{2}\right)$. In other words the relevant local micro-time scales are determined by the local strain rates such that $\tau_{\eta}=1 / \sqrt{2 \operatorname{tr}\left(\boldsymbol{s}^{2}\right)}$. The Probability Density Function (PDF) of all Stokes numbers $\tau_{p} / \tau_{\eta}$ in our flow is given in Fig. 2. The red solid, blue dashed-dotted and black dashed lines on this plot mark the values of $\tau_{p} / \tau_{\eta}$ which equal $\left\langle\tau_{p}\right\rangle /\left\langle\tau_{\eta}\right\rangle,\left\langle\tau_{p} / \tau_{\eta}\right\rangle$ and $\left\langle\tau_{p}\right\rangle / \bar{\tau}_{\eta}$ respectively (the brackets being averages over all particles or all space accordingly). We checked that the PDF of $\tau_{p} / \tau_{\eta}$ is very similar to the PDF of $1 / \tau_{\eta}$ as expected from the fact that the PDF of $\tau_{p}$ is very narrowly peaked, and we also checked that the PDF of $\operatorname{tr}\left(s^{2}\right)$ is peaked at zero, which agrees with the observation that the PDFs of $\tau_{p} / \tau_{\eta}$ and of $1 / \tau_{\eta}$ both vanish at zero. Finally, we verified that the PDFs of partial derivatives of velocity components with respect to spatial coordinates are approximate gaussians peaked at 0 .

Following Reade \& Collins [14], droplets are modelled as very small but dense circular inertial particles subjected to Stokes drag and impulsive forces $\boldsymbol{F}_{p}^{i j}$ resulting from collisions between $i$ th and the $j$ th particles. These particles are in fact point particles in the simulations evolving according to

$$
\begin{gathered}
\frac{\mathrm{d} \boldsymbol{x}_{p}^{i}}{\mathrm{~d} t}=\boldsymbol{v}_{p}^{i} \\
\frac{\mathrm{d} \boldsymbol{v}_{p}^{i}}{\mathrm{~d} t}=\frac{1}{\tau_{p}^{i}}\left(\boldsymbol{u}\left(\boldsymbol{x}_{p}^{i}, t\right)-\boldsymbol{v}_{p}^{i}\right)+\frac{1}{m_{p}^{i}} \sum_{j \neq i} \boldsymbol{F}_{p}^{i j}
\end{gathered}
$$

where $\boldsymbol{x}_{p}^{i}, \boldsymbol{v}_{p}^{i}, m_{p}^{i}$ and $\tau_{p}^{i}=\frac{2}{9} \frac{\rho_{p}}{\rho_{f}} \frac{\left(a_{p}^{i}\right)^{2}}{\nu}=\frac{2 \rho_{p}}{9 \rho_{f}}\left(\frac{a_{p}^{i}}{\eta}\right)^{2} \bar{\tau}_{\eta}$ are the positions, velocities, masses and response times of particles of radius $a_{p}^{i}$, and $\boldsymbol{u}\left(\boldsymbol{x}_{p}^{i}, t\right)$ are the carrier fluid velocities at $\boldsymbol{x}_{p}^{i}(t)$ at time $t$.

A method based on molecular-dynamic-simulation strategies [15, 20] checks for collisions between particles. Collisions are enacted in a random order whenever two

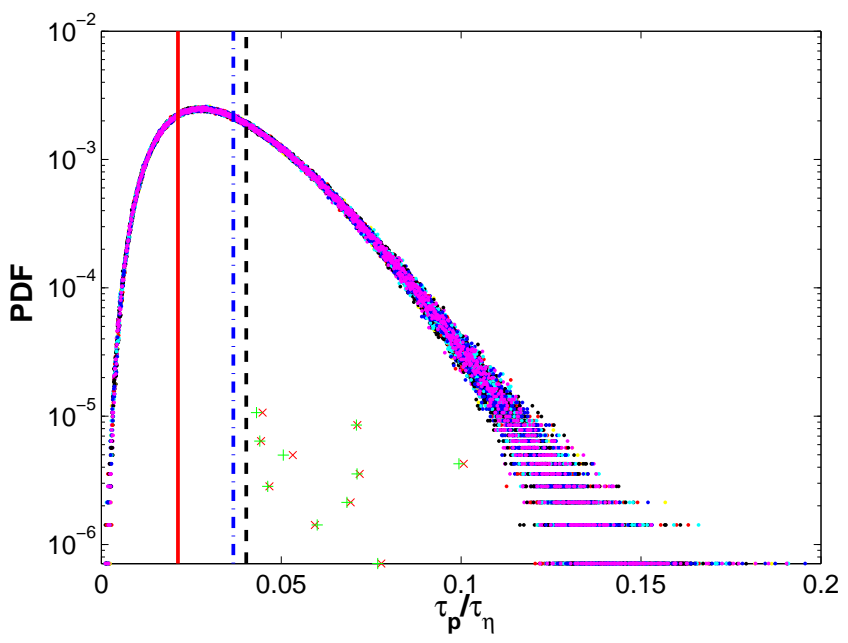

FIG. 2: (Color online) PDFs of $\tau_{p} / \tau_{\eta}$ at $t=0$ and at the times of the first 10 collisions for the case where $\phi_{A}=10^{-4}$ and $\eta /\left(2 \pi / k_{c}\right)=1 / 5$, see Table凹. The red solid, blue dasheddotted and black dashed lines indicate where $\left\langle\tau_{p}\right\rangle /\left\langle\tau_{\eta}\right\rangle=$ $2.13 \cdot 10^{-2},\left\langle\tau_{p} / \tau_{\eta}\right\rangle=3.67 \cdot 10^{-2}$ and $\left\langle\tau_{p}\right\rangle / \bar{\tau}_{\eta}=4.03 \cdot 10^{-2}$ are, respectively, on the plot. The pairs of red crosses and green plus signs on the plot correspond to early collisions and indicate, on the abscissa, the values of the local Stokes numbers $\tau_{p} / \tau_{\eta}$ of the two colliding particles one time step before collision. The ordinate value of these crosses and plus signs is arbitrary except for the order of collisions, the red cross and the green plus sign corresponding to the first (tenth) collision having the lowest (highest) ordinate value. The fact that crosses and plus signs for the same collision are always very close to each other reflects the fact that the first ten collisions are for similar-size particles.

point particles get so close that

$$
\left\|\boldsymbol{x}_{p}^{i}-\boldsymbol{x}_{p}^{j}\right\| \leq\left(a_{p}^{i}+a_{p}^{j}\right)
$$

It is commonly accepted that the coalescence efficiency is close to one for droplets with radius less than $100 \mu \mathrm{m}$ because their ratios of inertial force to surface tension are small [21]. However, collision efficiencies may be estimated to be of the order of a few tens of percent for droplets of $15 \mu \mathrm{m}$ radius or so [22, 23] even if we take into account turbulent enhancement of these efficiencies 21. Nevertheless, as an initial simplifying assumption, we take collision efficiencies to be 1 in this study. All our collisions are therefore assumed to give rise to a coagula- 
tion event thus resulting in a new circular particle with the following properties

$$
\begin{gathered}
m_{p}^{i, \text { new }}=m_{p}^{i}+m_{p}^{j} \\
\boldsymbol{v}_{p}^{i, \text { new }}=\left(m_{p}^{i} \boldsymbol{v}_{p}^{i}+m_{p}^{j} \boldsymbol{v}_{p}^{j}\right) / m_{p}^{i, \text { new }} .
\end{gathered}
$$

The positions and velocities of the remaining (noncolliding) particles are then advanced using 4th-order Runge-Kutta algorithm. To ensure accurate integrations of particle trajectories, the timestep $\Delta t$ used in our DNS satisfies $\Delta t \ll \min _{i}\left(\tau_{p}^{i}\right) \ll \eta / u^{\prime}<\bar{\tau}_{\eta}<\left\langle\tau_{\eta}\right\rangle$. This sets a lower bound to our choice of $\eta$ and therefore a lower bound to the volume fraction $\phi_{V}$ which we can emulate via equation (1) for a certain number $N_{2}$ of initial droplets. Indeed, $\frac{L_{b o x}}{\left\langle a_{p}\right\rangle}=\frac{L_{b o x}}{L} \frac{L}{2 \pi / k_{c}} \frac{2 \pi / k_{c}}{\eta} \frac{\eta}{\left\langle a_{p}\right\rangle}$, and in our simulations $\frac{L_{b o x}}{L}=\frac{2 \pi}{0.245}=25.65, \frac{L}{2 \pi / k_{c}} \approx 22.18$ and $\frac{\eta}{\left\langle a_{p}\right\rangle} \approx 74.1$ for the initial distribution of droplet sizes. For equation (1) to give us a realistic volume fraction $\phi_{V} \sim 10^{-6}$ would require either extremely small initial $N_{2}$ or extremely large $\frac{2 \pi / k_{c}}{\eta}$ which our time stepping does not allow. (A very large $\frac{2 \pi / k_{c}}{\eta}$ would also require a very much larger DNS with a very much wider range of excited scales to be justified.) Furthermore, very small initial values of $N_{2}$ require much longer integration times than we can afford. We have therefore opted for the following three sets of simulations: $N_{2} \approx 1.5 \cdot 10^{6}$ and $\eta /\left(2 \pi / k_{c}\right)=1 / 5$ (case of Table I); $N_{2} \approx 5.5 \cdot 10^{5}$ and $\eta /\left(2 \pi / k_{c}\right)=1 / 10 ; N_{2} \approx 1.25 \cdot 10^{5}$ and $\eta /\left(2 \pi / k_{c}\right)=1 / 15$. In the first case, equation (1) gives $\phi_{V} \approx 10^{-3}$, and in the second and third cases equation (1) gives $\phi_{V} \approx 2 \cdot 10^{-4}$ and $\phi_{V} \approx 3 \cdot 10^{-5}$ respectively. Hence, we always overestimate typical volume fractions in warm clouds by two to three orders of magnitude, but we also underestimate the turbulence intensity by a factor of 10. Defining an a priori frequency of collisions as $u^{\prime} / L_{b o x}$ times the geometrical probability of interception introduced in the text leading to equation (1), this a priori frequency is $f_{2} \equiv \frac{u^{\prime}}{L_{b o x}} \frac{12 a_{p}}{\pi l_{2}}$ in our simulations and, for the case corresponding to $\phi_{V}=10^{-3}$, turns out to be 10 times smaller than in 3D warm cloud conditions where the volume fraction is $\phi_{V}=10^{-6}$ and the turbulence intensity is 10 times larger than here, i.e. $f_{2} \approx 10 f_{3}^{w c}$.

In the case where $\eta /\left(2 \pi / k_{c}\right)=1 / 5$ and $\phi_{V}=10^{-3}$, we integrate the trajectories of about 1.5 million initial particles for 22 outer time-scales $\tau_{L} \equiv L / u^{\prime}$ of our flow. Based on the parameters of Table \which are for this case, and assuming that $\eta$ is about $1 \mathrm{~mm}$ and $\bar{\tau}_{\eta}$ is about $0.1 \mathrm{~s}$, this total integration time corresponds to less than a minute, which is extremely short compared to the typical fifteen minutes to half hour usually required for droplets to grow from about $15 \mu \mathrm{m}$ to about $50 \mu \mathrm{m}$ in warm clouds. Note that $22 \tau_{L} f_{2}$ is of the same order as fifteen minutes multiplied by $f_{3}^{w c}$.

Numbers of collisions as a function of time are plotted in Fig. 3. We consider two different types of initial conditions. One where the point particles are randomly distributed, in which case collisions occur immediately after $t=0$ because a sizeable number of point particles find themselves, initially, close enough for (4) to hold. And one where the point particles are distributed on a regular lattice so that (4) does not hold initially for all pairs of particles. It might be interesting to note (see Fig. (3) that, whilst the initial evolution of the number of collisions is very different in these two cases, they converge towards a similar time dependence at large enough times $t / \tau_{L}=t u^{\prime} / L$.

In the case where particles are uniformly distributed at $t=0$, the first collision does not occur immediately, but at a time equal to about $5.1 \bar{\tau}_{\eta} \approx 2.67\left\langle\tau_{\eta}\right\rangle$. This collision is between particles that have a local Stokes number $\tau_{p} / \tau_{\eta}$ which is between 2 and 4 times larger than the average Stokes number, depending on the way one choses to estimate it, see Fig. 2, All ten first collisions occur between time $t=5.1 \bar{\tau}_{\eta} \approx 2.67\left\langle\tau_{\eta}\right\rangle$ and time $t=8.8 \bar{\tau}_{\eta} \approx 4.61\left\langle\tau_{\eta}\right\rangle$ after $t=0$ and involve pairs of particles with Stokes numbers well above any estimate of the average Stokes number. They therefore occur within about a second and are caused by extreme events within the air turbulence where, locally, the Stokes number is higher than average. Considerations based only on the average Stokes number would miss these collisions and would therefore also miss the process initiating droplet size growth. Note, in particular, that the local Stokes number characterising the first and fifth collisions are respectively 0.08 and 0.1 , just about large enough for meagre signs of clustering to be present if the average Stokes number had such a value (see Fig. 1 in [16] and Fig. 1 in [17]).
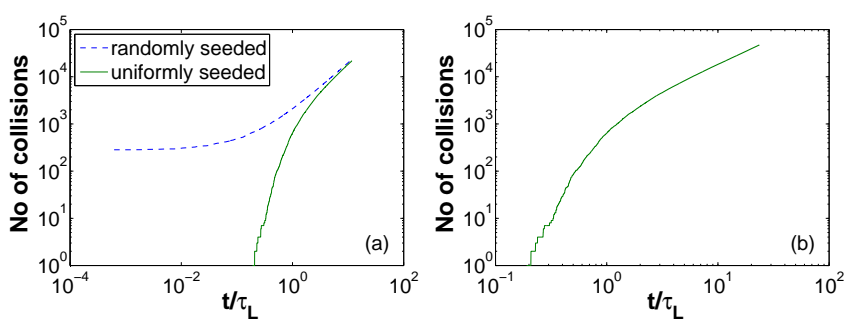

FIG. 3: (Color online) Numbers of collisions of pairs of particles as functions of $t / \tau_{L}=t u^{\prime} / L$ for the case where $\phi_{A}=10^{-4}$ (see Table I). (a) Evolution up to $t / \tau_{L}=11$ for two different initial distributions of particles. (b) Evolution up to $t / \tau_{L}=22$ for the initially uniform distribution of particles.

Figure 4 4 shows PDFs of particle radii at different times. At $t=0$, this PDF is very sharply peaked around $0.0135 \eta$. By $t=3 \tau_{L}$ a second peak has appeared at a value about 1.4 times larger than $0.0135 \eta$, and as time progresses more peaks appear at progressively higher values of the particle radius. By the end of the simulation, i.e. when $t / \tau_{L}=22$, the largest particle has a radius nearly three times larger than the initial particle 
radii. This result of our simulations is noteworthy because $t / \tau_{L}=22$ corresponds to about less than a minute and the factor 3 is not too far from the ratio of $50 \mu \mathrm{m}$ to $15 \mu \mathrm{m}$.
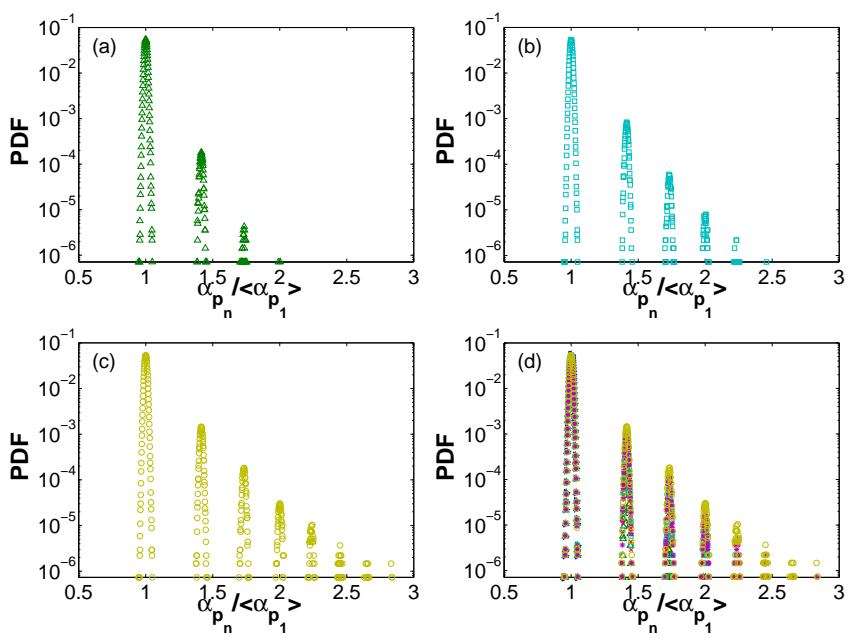

FIG. 4: (Color online) PDFs of the particle radius $a_{p}$ normalised by the initial average particle radius $\left\langle a_{p}\right\rangle$ at different times; in plot (a) time $3 \tau_{L}$, (b) time $11.6 \tau_{L}$, (c) time $22 \tau_{L}$. In plot (d), six PDFs are plotted together at six different times: blue $\triangleright 0.0 \tau_{L}$, green $\triangle 3.0 \tau_{L}$, red $\times 8.5 \tau_{L}$, cyan $\square 11.6 \tau_{L}$, magenta $* 17.0 \tau_{L}$, yellow $\circ 22 \tau_{L}$.

In conclusion, our calculations suggest that turbulence in air which is carrying water droplets so small that their average Stoker number is of order $10^{-2}$ and therefore too small to significantly cluster can nevertheless initiate a process of droplet growth via collisions and coalescence which is fast enough for droplets to grow from $15 \mu \mathrm{m}$ to $50 \mu \mathrm{m}$ within about a minute. The reason behind this fast growth is in the wide spread of local Stokes numbers in a turbulent flow. A few local flow events exist in the turbulent field where the local Stokes number is much higher than the average Stokes number and high enough to cause a few pairs of droplets to collide and coalesce quite quickly even if small-scale intermittency and differential gravitational settling are not taken into account. However, this initiation mechanism may not be effective if the volume fraction is too low. When we took the simulated volume fraction $\phi_{V}$ to be smaller than $10^{-3}$, i.e. $\phi_{V} \approx 2 \cdot 10^{-4}$ and $\phi_{V} \approx 3 \cdot 10^{-5}$, the first ten collisions returned by our simulations were at significantly later times and not all local Stokes numbers in these collisions were significantly larger than average (6 and 3 pairs, respectively, in the $\phi_{V} \approx 2 \cdot 10^{-4}$ and $\phi_{V} \approx 3 \cdot 10^{-5}$ cases $)$. Nevertheless, we stress that we have found a turbulence-generated droplet growth phenomenon which takes droplets from $15 \mu \mathrm{m}$ to $50 \mu \mathrm{m}$ within a time which, if multiplied by our estimated a priori frequency of collisions, is comparable to the time required for droplets to grow from $15 \mu \mathrm{m}$ to $50 \mu \mathrm{m}$ in warm clouds.
Our results support Kostinski's \& Shaw's [12] suggestion that powerful rare events can cause initiation of fast droplet growth by turbulence. However, our results also suggest that this phenomenon may not require smallscale intermittency and/or differential gravitational settling if collision efficiency is assumed perfect. Nevertheless, one can surely expect selectively enhanced settling velocities [13] and/or small-scale intermittency to increase the number and/or power of events which can accelerate average growth rate and thereby perhaps outweigh the adverse effect of collision efficiencies which are typically one order of magnitude smaller than assumed in this work.

The authors are grateful to Ryo Onishi, Wojciech Grabowski and Bernhard Mehlig for reading the first version of this manuscript and making very helpful suggestions and comments.

[1] R. A. Shaw, Ann. Rev. Fluid Mech. 35, 183 (2003).

[2] M. B. Baker, Science 276, 1072 (1997).

[3] L.-P. Wang, Y. Xue, O. Ayala, and W. Grabowski, Atmos. Res. 83, 416 (2006).

[4] G. Falkovich and A. Pumir, J. Atmos. Sci. 64, 4497 (2007).

[5] L.-P. Wang, A. Wexler, and Y. Zhou, J. Fluid Mech. 415, $117(2000)$.

[6] S. Sundaram and L. R. Collins, J. Fluid Mech. 335, 75 (1997).

[7] Y. Zhou, A. S. Wexler, and L.-P. Wang, Phys. Fluids 10, 1206 (1998).

[8] M. Wilkinson, B. Mehlig, and V. Bezuglyy, Phys. Rev. Lett. 97, 048501 (2006).

[9] P. A. Vaillancourt and M. K. Yau, Bull. Amer. Meteor. Soc. 81, 285 (2000).

[10] P. G. Saffman and J. S. Turner, J. Fluid Mech. 1, 16 (1956).

[11] S. B. Pope, Turbulent Flows (Cambridge University Press, 2000).

[12] A. B. Kostinski and R. A. Shaw, Bull. Amer. Meteor. Soc. 86, 235 (2005).

[13] S. Ghosh, J. Dávila, J. C. R. Hunt, A. Srdic, H. J. S. Fernando, and P. R. Jonas, Proc. R. Soc. A 461, 3059 (2005).

[14] W. C. Reade and L. R. Collins, J. Fluid Mech. 415, 45 (2000).

[15] S. Sundaram and L. R. Collins, J. Comput. Phys. 124, 337 (1996).

[16] W. C. Reade and L. R. Collins, Phys. Fluids 12, 2530 (2000).

[17] L. Chen, S. Goto, and J. C. Vassilicos, J. Fluid Mech. 553, 143 (2006).

[18] P. Tabeling, Phys. Rep. 362, 1 (2002).

[19] S. Goto and J. C. Vassilicos, New J. Phys. 6, 65 (2004).

[20] M. P. Allen and D. J. Tildesley, Computer simulation of liquids (Oxford University Press, 1987).

[21] L.-P. Wang, O. Ayala, B. Rosa, and W. W. Grabowski, New J. Phys. 10, 075013 (2008).

[22] P. R. Jonas, Atmos. Res. 40, 283 (1996). 
[23] H. R. Pruppacher and J. D. Klett, Microphysics of clouds and precipitation (Kluwer Academic Publishers, 1998). 